\title{
Dielectric Relaxation, Conductivity Mechanism and Complex Impedance Spectroscopic Studies of Pure and Cadmium Mixed Cobalt Levo-Tartrate Crystals
}

\author{
N.H. Manani ${ }^{1}$, H.O. Jethva ${ }^{2^{*}}$, M.J. Joshi ${ }^{3}$ \\ ${ }^{1}$ M. S. M. Science College, Morbi, Gujarat, India \\ ${ }^{2,3}$ Department of Physics, Saurashtra University, Rajkot, Gujarat, India \\ *Corresponding Author: hojethva@rediffmail.com, Tel.- 9426237404
}

Available online at: www.isroset.org

Received: 10/Jan/2020, Accepted: 10/Feb/2020, Online: 28/Feb/2020

\begin{abstract}
Cadmium tartrate is known for piezoelectric application and cobalt tartrate finds application in electrochemical depositions. Therefore, in the present study, an attempt is made to grow mixed levo-tartrates of cobalt and cadmium. The pure and mixed levo-tartrate crystals of cobalt and cadmium with different volume concentration of cobalt nitrate and cadmium nitrate solutions are grown by using gel growth technique. Brownish spherulitic crystals are grown. The EDAX study confirms the exact composition of cobalt and cadmium in the grown crystals. The impedance spectroscopic studies are carried out on pelletized samples from $100 \mathrm{~Hz}$ to $1 \mathrm{MHz}$ frequency range at room temperature. The Nyquist plots exhibit one semicircle for pure and mixed crystals of cobalt and cadmium levo-tartrate due to grain contribution only. An attempt is made to provide equivalent R-CPE network for the grain contribution. The dielectric constant and dielectric loss decreases with increase in frequency. The Jonscher's power law is studied for ac conductivity and applied to the conductivity phenomena in pure and mixed crystals of cobalt and cadmium levo-tartrate. From the detailed impedance and dielectric analysis, it is found that different ac electrical and dielectric parameters are sensitive to the addition of cadmium and its concentration in cobalt levo-tartrate crystals.
\end{abstract}

Keywords - ac conductivity, Cobalt cadmium mixed levo-tartrate crystals, dielectric study, gel growth, impedance spectroscopy, Jonscher's law.

\section{INRODUCTION}

Tartrate crystal of cadmium is well known for its piezoelectric application [1] and carbonate solutions containing tartrate complexes of cobalt (II) find application in an electrochemical procedure of anodic deposition of cobalt oxyhydroxide film on a glassy carbon substrate in an alkali medium [2]. Earlier, the reports are available on structural, thermal, magnetic and dielectric studies of cobalt tartrate crystals $[3,4]$. Whereas, several studies like structural, thermal, FTIR, UV-Vis, electrical conductivity, optical absorption, micromechanical and dielectric studies are reported for cadmium tartrate crystals [5-8].

Since, the detailed analysis on dielectric relaxation, conductivity mechanism and complex impedance spectroscopic studies of pure cobalt levo-tartrate crystals and the effect of addition of cadmium do not exist in the literature, in the present paper, the authors report the gel growth of pure and mixed levo-tartrate crystals of cobalt and cadmium and investigation of the complex impedance spectroscopy and dielectric spectroscopy at room temperature within the frequency range $100 \mathrm{~Hz}$ to $1 \mathrm{MHz}$.

\section{EXPERIMENTAL}

In the present study, the single diffusion gel growth technique was employed to grow the mixed crystals of cobalt and cadmium levo-tartrate. The growth medium was silica hydro gel, which was set by mixing sodium metasilicate solution of specific gravity 1.05 with $1 \mathrm{M}$ levo tartaric acid solution in such a manner that the $\mathrm{pH}$ of the mixture was obtained 4.5. This solution was poured in test tubes of $150 \mathrm{~mm}$ length and $25 \mathrm{~mm}$ diameter to set in the gel form. The supernatant solution contained the volume compositions of cobalt nitrate and cadmium nitrate solutions mentioned in table 1 .

Table 1. Volume compositions of supernatant solution

\begin{tabular}{|l|l|l|l|l|}
\hline \multirow{2}{*}{$\begin{array}{l}\text { Sample } \\
\text { No }\end{array}$} & \multicolumn{4}{|l|}{ Name of supernatant solution } \\
\cline { 2 - 5 } & $\mathrm{Co}\left(\mathrm{NO}_{3}\right)_{2} \cdot 6 \mathrm{H}_{2} \mathrm{O}$ & \multicolumn{2}{l|}{$\mathrm{Cd}\left(\mathrm{NO}_{3}\right)_{2} \cdot 4 \mathrm{H}_{2} \mathrm{O}$} \\
\cline { 2 - 5 } & Volume & $\mathrm{Mol}$ & volume & Mol \\
\hline 1 & $10 \mathrm{ml}$ & $1 \mathrm{M}$ & $00 \mathrm{ml}$ & $00 \mathrm{M}$ \\
\hline 2 & $8 \mathrm{ml}$ & $1 \mathrm{M}$ & $2 \mathrm{ml}$ & $1 \mathrm{M}$ \\
\hline 3 & $6 \mathrm{ml}$ & $1 \mathrm{M}$ & $4 \mathrm{ml}$ & $1 \mathrm{M}$ \\
\hline 4 & $4 \mathrm{ml}$ & $1 \mathrm{M}$ & $6 \mathrm{ml}$ & $1 \mathrm{M}$ \\
\hline 5 & $2 \mathrm{ml}$ & $1 \mathrm{M}$ & $8 \mathrm{ml}$ & $1 \mathrm{M}$ \\
\hline
\end{tabular}


All the chemicals were of AR grade and obtained from Sigma Aldrich. The following reaction was expected to occur in the formation of cobalt cadmium mixed levotartrate crystals.

$(1-\mathrm{X}) \mathrm{Co}\left(\mathrm{NO}_{3}\right)_{2} \cdot 6 \mathrm{H}_{2} \mathrm{O}_{(\mathrm{aq})}+\mathrm{XCd}\left(\mathrm{NO}_{3}\right)_{2} \cdot 4 \mathrm{H}_{2} \mathrm{O}_{(\mathrm{aq})}+$ $\mathrm{H}_{2} \mathrm{C}_{4} \mathrm{H}_{4} \mathrm{O}_{6}+\mathrm{nH}_{2} \mathrm{O}=$

$\mathrm{Cd}_{\mathrm{X}} \mathrm{Co}_{(1-\mathrm{X})} \mathrm{C}_{4} \mathrm{H}_{4} \mathrm{O}_{6} \mathrm{nH}_{2} \mathrm{O}+4 \mathrm{HNO}_{3}+9 \mathrm{H}_{2} \mathrm{O}+\mathrm{O}_{2}$

Where, the value of $\mathrm{X}$ is to be determined from the EDAX analysis exactly. The amount of $\mathrm{HNO}_{3}$ produced is very less compared to the nutrients being supplied to the growing crystals and hence, no major limitations are imposed to the growth of crystals $[9,10]$.

Figures $1(a-d)$ are the photographs of the crystals growing inside the test tubes for the supernatant solutions (a), (b), (c), and (d), respectively.

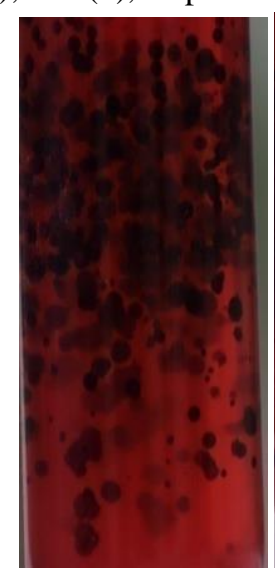

1(a)

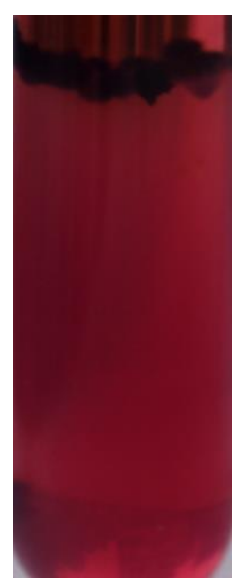

$1(\mathrm{~d})$

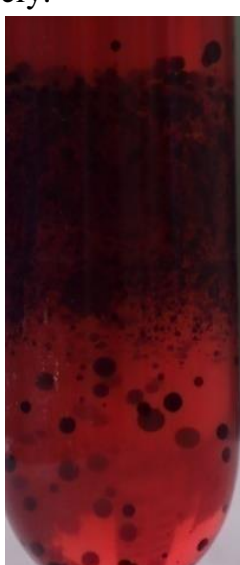

1(b)

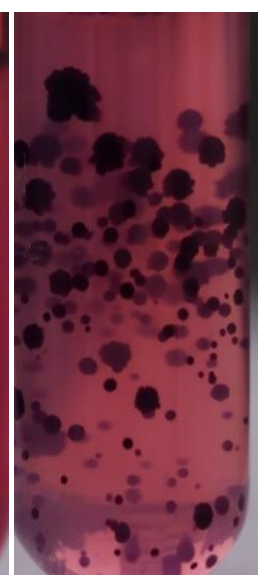

$1(\mathrm{e})$
Figure 1. Growth of crystals in test tubes

It is observed that brownish spherulitic crystals are grown, the number density of which are changed as per the composition of the supernatant solutions.

\section{RESULT AND DISCUSSION}

The determination of metallic elements in the crystals was carried by the EDAX using Philips X1 - 30. It is found that 77.66 mass \% Co and 22.34 mass\% Cd (sample-2), 43.68 mass \% Co and 56.32 mass $\%$ Cd (sample-3), 31.65 mass $\%$
Co and 68.35 mass\% Cd (sample-4) and 15.98 mass\% Co and 84.02 mass \% Cd (sample-2) were present in the mixed crystals of cobalt and cadmium levo-tartrate. In the mixed levo-tartrate crystals of cobalt and cadmium of samples 3, 4 and 5, the weight\% of cobalt is less compared to cadmium, which is due to the higher hydrated radius of cobalt compared to cadmium. In sample 2, the weight $\%$ of cadmium is less compared to cobalt, which is due to the lowest concentration of $\mathrm{Cd}^{+2}$ ions compared to $\mathrm{Co}^{+2}$ ions in the solution and less tendency to form compound of $\mathrm{Cd}^{+2}$ ions compared to $\mathrm{Co}^{+2}$ ions in the solution.

\section{Impedance spectroscopy}

Figure 2 shows the complex impedance spectrum, i.e. Nyquist plot at room temperature for samples 1 to 5 over a frequency range $100 \mathrm{~Hz}$ to $1 \mathrm{MHz}$. The Nyquist plot of all the samples 1 to 5 consists of one region over the whole frequency range, indicating grain effect only within the range of frequency studied. The effect of addition of cadmium in different proportion on impedance characteristics of pure cobalt levo-tartrate sample 1 is clearly visible in the figure. The impedance property of the material under study is characterized by the appearance of semicircular arcs whose pattern gradually changes with the change in wt $\%$ of cadmium. Such patterns, when modeled in terms of an equivalent electrical circuit, provides useful information related to electrical processes occurring within the material under study and their correlation with microstructure.

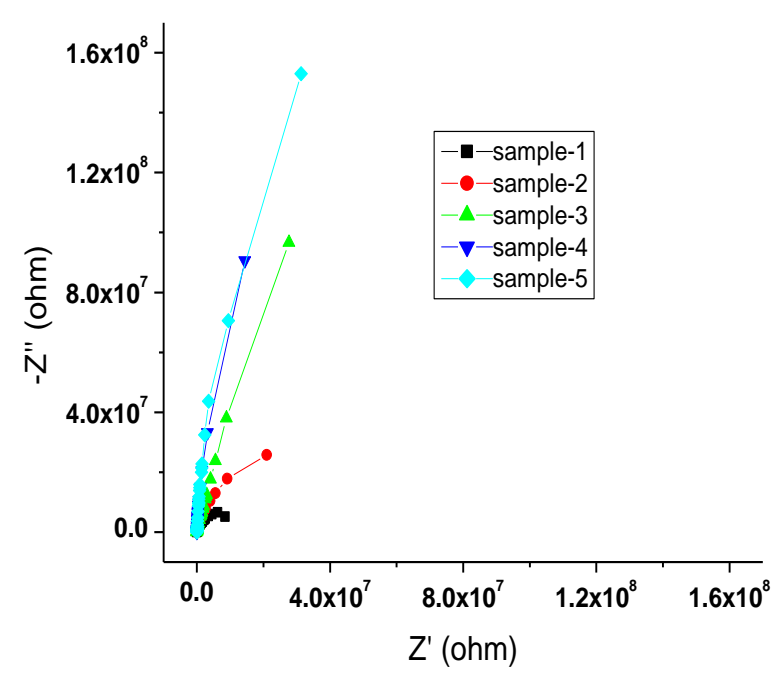

Figure 2. Nyquist plots of samples 1 to 5

In the present case, when the Nyquist plot of all the samples 1 to 5 is fitted by using the software Z-view, it is observed that all the Nyquist plot consist of one region over the whole frequency range, indicating grain effect only. Generally, the semicircular arcs of the impedance pattern can be assigned to a parallel combination of resistance and capacitance. But in the present case, the presence of grain is modeled using parallel R-CPE circuit for all the samples 1 to 5 and it is shown in the figure 3 . 


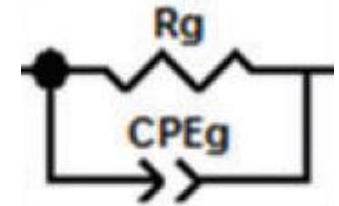

Figure 3. R-CPE circuit

Instead of capacitance, the CPE, i.e. Constant Phase Element is introduced in the circuit due to non-ideal capacitive behavior. The capacitance values for all the samples 1 to 5 are calculated by using the equation $\mathrm{C}=$ $\left(\mathrm{R}^{(1-\alpha)} \mathrm{Q}\right)^{1-\alpha}[11]$. Here, $\alpha$ is the degree of deviation with respect to the value of the pure capacitor. Its value is unity for the pure capacitor and is zero for pure resistor. As only grain effect is dominant in all the samples within the frequency range studied, $\mathrm{R}$ represents the grain resistance, the value of which can be obtained from the intercept of each semicircle with real axis. It can be noticed from the Nyquist plots of figure 2 that the smallest semicircle is obtained for the pure crystals of cobalt levo-tartrate of sample 1 . Then as the wt $\%$ of cobalt is decreased and cadmium is decreased in moving from sample 2 to 5 , the diameter of the semicircle progressively increases with a shift of the centre away from the origin of the plot. These indicate increase in the value of grain resistance and accordingly, decrease in the value of conductivity of the samples. The values of the grain resistance and capacitance are summarized in Table 2. The relaxation time for grain for each sample was calculated by using the relation $\tau_{\mathrm{g}}=\mathrm{R}_{\mathrm{g}} \cdot \mathrm{C}_{\mathrm{g}}$. The values of $\alpha_{\mathrm{g}}$ show the degree of deviation for grain. Both the values are listed in the table 2 for all the samples 1 to 5 .

Table 2. Equivalent circuit parameters of samples 1 to 5

\begin{tabular}{|l|l|l|l|l|}
\hline $\begin{array}{l}\text { Sample } \\
\text { No }\end{array}$ & $\mathrm{R}_{\mathrm{g}}(\mathrm{M} \Omega)$ & $\mathrm{C}_{\mathrm{g}}(\mathrm{pF})$ & $\alpha_{\mathrm{g}}$ & $\tau_{\mathrm{g}}(\mathrm{ms})$ \\
\hline 1 & 14.15 & 24.4 & 0.904 & 0.345 \\
\hline 2 & 42.5 & 18.0 & 0.930 & 0.765 \\
\hline 3 & 407 & 16.3 & 0.925 & 6.648 \\
\hline 4 & 395 & 15.6 & 0.983 & 6.171 \\
\hline 5 & 879 & 7.62 & 0.973 & 6.698 \\
\hline
\end{tabular}

From the table 2, it is observed that the grain relaxation time is more in cadmium added cobalt levo-tartrate crystals of samples 2 to 5 . This indicates that the presence of cadmium lowers the relaxation process of grain present in the samples. Further, the values of $\alpha_{\mathrm{g}}$ are high for cadmium added cobalt levo-tartrate crystals of samples 2 to 5. The increase of the values of $\alpha_{\mathrm{g}}$ for grain due to addition of cadmium in the pure cobalt levo-tartrate crystal shows that the defect or impurities in the grains increase due to addition of cadmium.

To recognize the relaxation process present within all the samples 1 to 5 clearly, the Bode plot is taken into account. Bode plots for all the samples 1 to 5 are presented in figure 4.

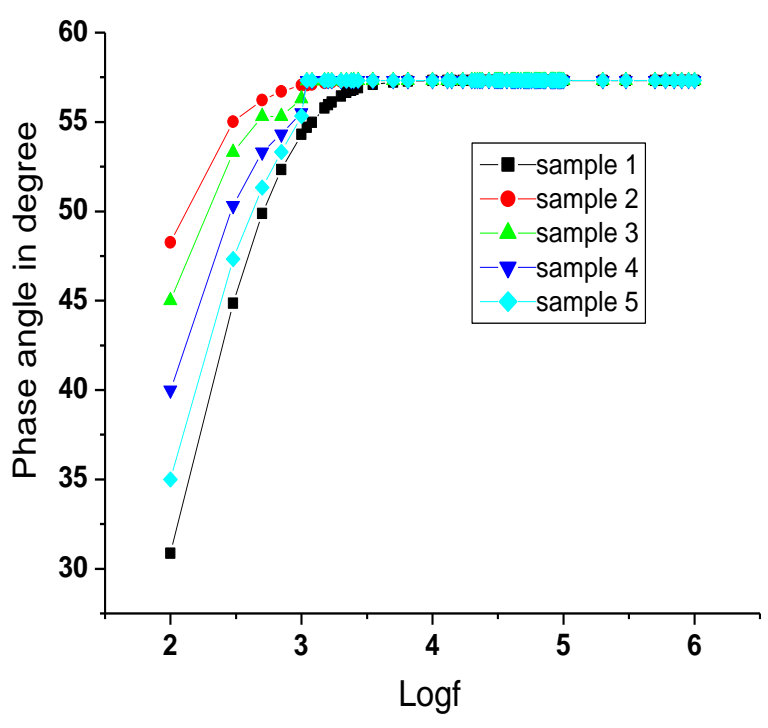

Figure 4. Bode plots of samples 1 to 5

The plots show the dependence of phase of the impedance on frequency. At lower frequencies and at higher frequencies, the observed shapes confirm the presence of grain only and relaxation mechanisms expressed by the equivalent R-CPE circuits [12].

Figure 5 shows the variation of the real part of complex impedance (Z') with respect to the applied frequency for all the samples 1 to 5 .

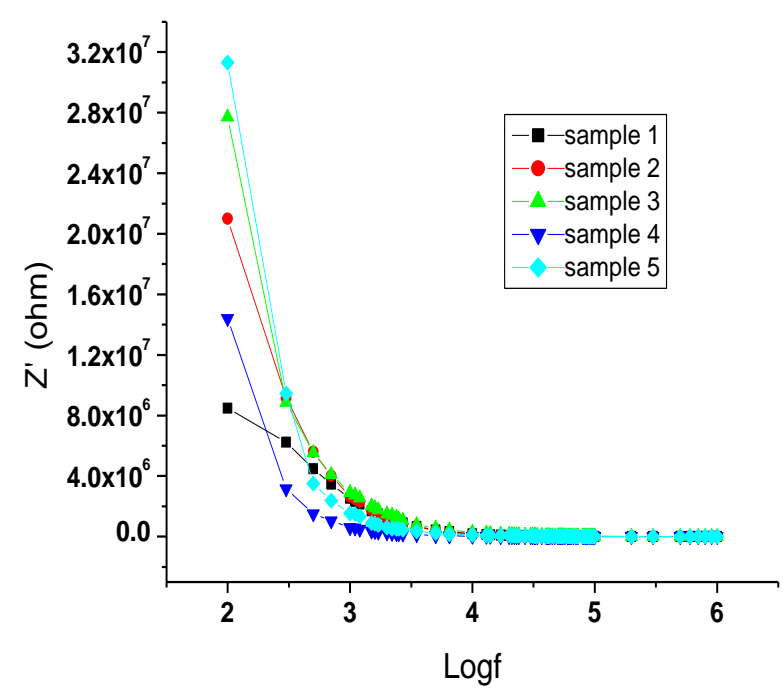

Figure 5. Z' versus logf curves of samples 1 to 5

It can be seen from the figure that the value of $Z$ ' decreases with increase in frequency for all the samples 1 to 5 , indicating the increase of ac conductivity. At higher frequencies, $Z$ ' achieves nearly a very low constant value, merge together, becomes constant and independent of frequency for all samples 1 to 5 . This nature may be due to the release of space charge [12]. The decrease in the value of Z' with increase in frequency at lower frequency region 
supports a slow dynamic relaxation process in all the samples 1 to 5 , probably due to space charge that gets released at higher frequencies [13]

Further, the lowest value of $Z^{\prime}$ at low frequency is obtained for pure cobalt levo-tartrate crystals of sample 1, which indicates the highest value of ac conductivity for that sample, As cadmium added into the pure sample 1, the value of $Z$ ' increases, which shows decrease in the value of ac conductivity for the cadmium added cobalt levotartrate crystals of samples 2 to 5 . A particular frequency at which Z' becomes independent of frequency is observed to shift towards the lower frequency side after addition of cadmium into the pure sample 1 of cobalt levo-tartrate crystals. This shifting in $Z$ ' plateau towards lower frequency side indicates the existence of frequency relaxation process in the samples [14] and in good agreement with the increase in grain relaxation time $\tau_{\mathrm{g}}$ as mentioned in table 2 .

Figure 6 shows the variation of imaginary part of complex impedance (Z') with respect to applied frequency for all the samples 1 to 5 .

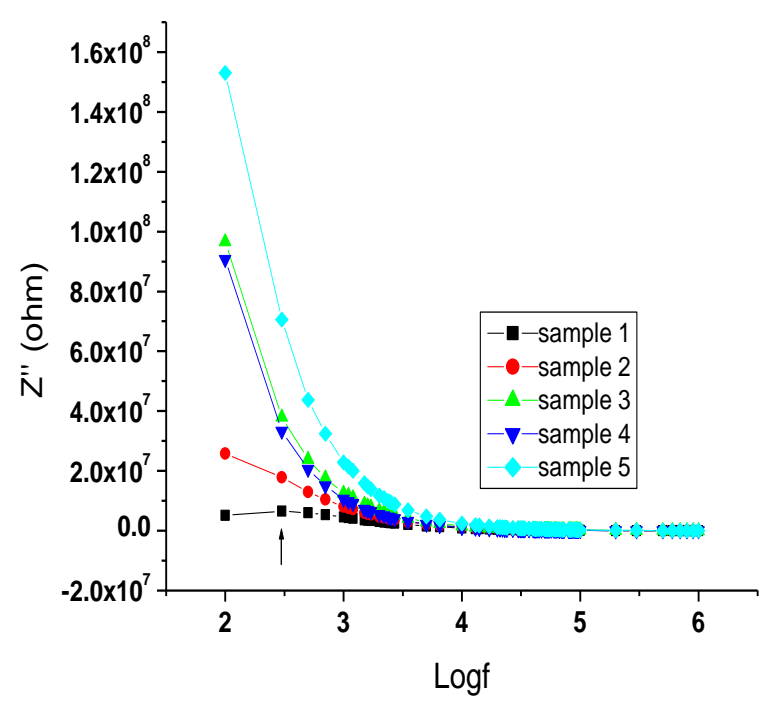

Figure 6. Z"' versus logf curves of samples 1 to 5

In the low frequency region, variation in $Z$ " for cadmium added cobalt levo-tartrate samples 2 to 5 shows behavior similar to Z'. This may be due to presence of space charge polarization at lower frequencies and its elimination at higher frequencies [13]. In the case of pure cobalt levotartrate crystals of sample $1, Z$ " shows a small peak in the lower frequency region, which is shown by an arrow in the figure 6. When cadmium is added, the peak is not observed (samples 2 to 5) and it might be shifted towards lower frequency region with increasing height. However, the lower frequency region may be beyond the frequency measurement under study to observe this phenomena. The increase in the peak height of cadmium added cobalt levotartrate crystals of samples 2 to 5 implies that the impedance of the samples 2 to 5 increases and shifting of the peak towards lower frequency region implies that the grain relaxation time $\tau_{\mathrm{g}}$ also increases. Both the results are in good agreement with the results of grain resistance $\left(R_{g}\right)$ and relaxation time $\left(\tau_{\mathrm{g}}\right)$ mentioned in table 2 . Further, $\mathrm{Z}$, are independent of frequency and merge together at higher frequencies due to the release of space charge in all the samples [12].

\section{Dielectric spectroscopy}

Figure 7 shows the variation in dielectric constant as a function of frequency for all the samples 1 to 5 . The nature of the plots shows high value of dielectric constant at lower frequency region for all the samples, which can be considered as a normal dielectric behavior [15] in pure and mixed tartrate compounds [16-18] then it is decreased with increase in frequency.

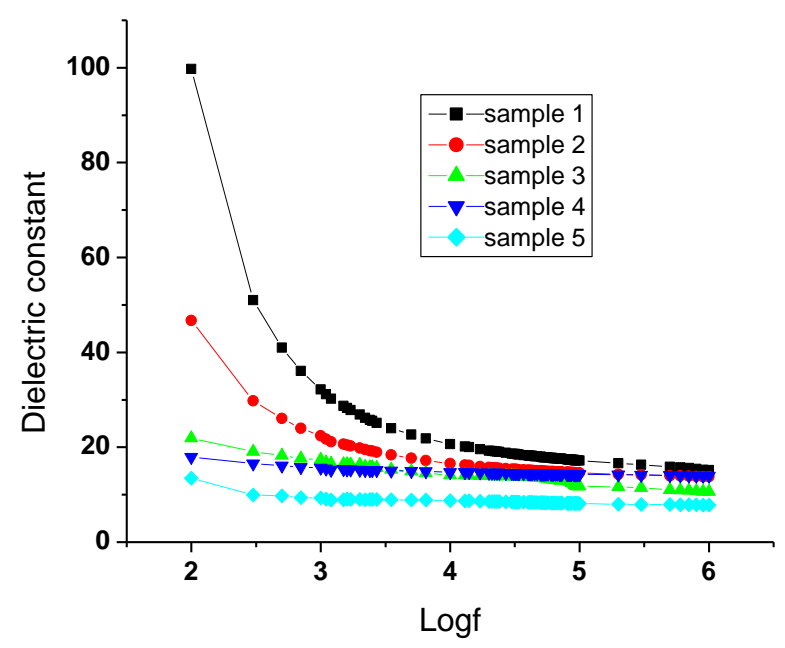

Figure 7. Dielectric constant versus logf

Further, the decrease in the value of dielectric constant with increasing frequency is in agreement with the Koops and Maxwell-Wagner models [19-21]. According to this model, the high value of dielectric constant at low frequencies can be attributed to the presence of all types of polarizations like electronic, ionic dipolar or orientational and space charge polarizations. As the frequency increases, the inadequacy of the dipoles to follow the applied ac electric field, results into the decrease in the value of dielectric constant as well as constant value of dielectric constant.

Further, it can be noticed that the magnitude of dielectric constant is lower for cadmium doped cobalt levo-tartrate crystals of samples 2 to 5. As cadmium content is increased on moving from sample 2 to 5 , the magnitude of dielectric constant decreases accordingly. This can be explained on the basis of relation between dielectric constant and capacitance. The dielectric constant is related with the capacitance by the relation $\mathrm{K}=\mathrm{Ct} / \varepsilon_{0} \mathrm{~A}$, where $\mathrm{C}$ is the capacitance, $\varepsilon_{0}$ is the permittivity of free space, $t$ and $A$ are the thickness and cross sectional area of the pellet, respectively. From table 2, it is observed that the highest value of grain capacitance is obtained for pure cobalt levotartrate crystals of sample 1 , which results into the highest value of dielectric constant. As cadmium is added, grain 
capacitance is decreased, which results into the decreased value of dielectric constant for cadmium added cobalt levo-tartrate crystals. As cadmium content is increased on moving from sample 2 to 5 , the grain capacitance decreases accordingly, this results into the decreased value of dielectric constant accordingly.

Figure 8 shows variation in dielectric loss as a function of frequency for all the samples 1 to 5 .

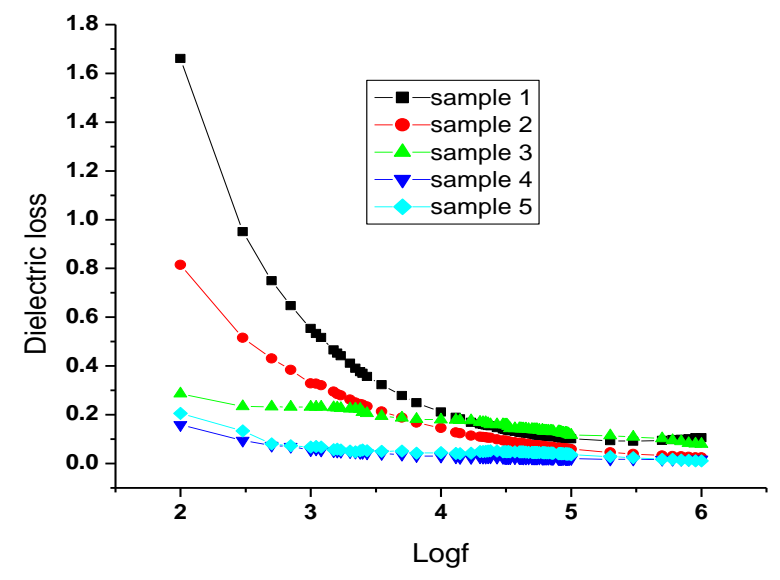

Figure 8. Dielectric loss versus logf

The dielectric loss curves are exhibiting the higher values for all the samples 1 to 5 at lower frequency and decreased gradually as frequency increases. The higher value of dielectric loss at lower frequency can be attributed to the presence of space charge polarization. The lower value of dielectric loss of cadmium added cobalt levo-tartrate crystals of samples 2 to 5 is due to the presence of cadmium at grain positions reducing the grain capacitance and ultimately reduces the dielectric loss.

\section{AC conductivity mechanism}

In the present study, the ac conductivity $\left(\sigma_{\mathrm{ac}}\right)$ value is calculated by using the expression $\sigma a c=\omega \varepsilon_{0} \varepsilon '$, where $\omega$ is the angular frequency, $\varepsilon_{0}$ is the permittivity of free space and $\varepsilon "$ is the dielectric loss. Figure 9 shows the ac conductivity variation with respect to frequency for all the samples 1 to 5 .

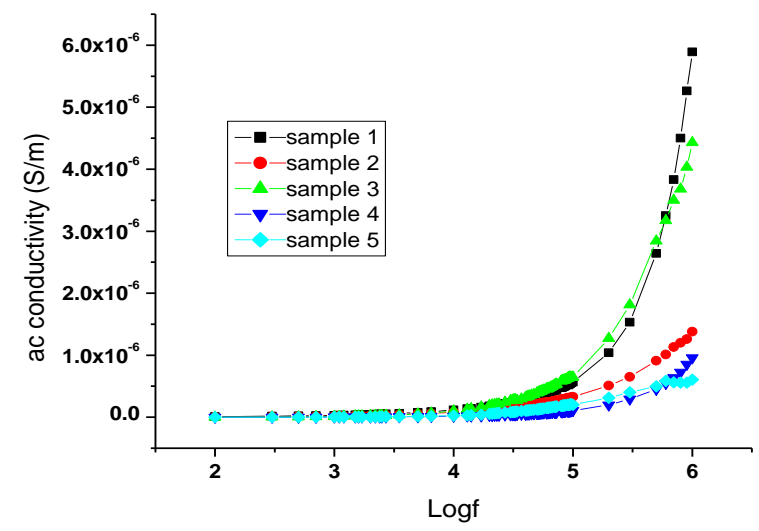

Figure 9. ac conductivity versus logf
It is observed from the figure 9 that the ac conductivity curves for all the samples 1 to 5 consist of two different regions within the measured frequency window limit (i) a low frequency flat or plateau region and (ii) a high frequency dispersion region. A low frequency plateau region in which conductivity remains frequency independent up to a certain frequency can be assigned to the dc conductivity of the samples, the value of which can be obtained by extrapolating the conductivity value to the lower frequency [22]. In this region where the conductivity is constant, the transportation of the ions takes place on infinite paths [23], resulting into the much faster travel of the ions and jump from one site to another available site.

The successful hoping of ions to a neighboring vacant site contributes to the dc conductivity [24]. A low frequency plateau region is followed by a high frequency dispersion region in which conductivity continuous to increase with increase in frequency. The frequency at the dispersion region deviated from the dc conductivity plateau is defined as characteristic frequency $\left(\omega_{\mathrm{p}}\right)$, also known as hopping rate. The careful observation of figure 9 shows that as the cadmium is added in to the pure cobalt levo-tartrate crystals of sample 1, the transition from frequency independent plateau region to the ac conductivity dispersion region shift towards the lower frequency side. Thus, the hopping rate $\left(\omega_{\mathrm{p}}\right)$, at which the relaxation effects begin to appear, decrease and move towards the lower frequency side. This result leads to the conclusion that lower the conductivity of the cadmium added cobalt levotartrate samples 2 to 5 compared to pure cobalt levotartrate sample 1, the higher is their relaxation time. This is in good agreement with the relaxation time given in table 2 and the dc conductivity values for all the samples 1 to 5 given in the table 3 .

The frequency dependence of conductivity or so called universal dynamic response of ionic conductivity can be related by an expression given by Jonscher's power law

$[25], \sigma_{\text {total }}(\omega, T)=\sigma_{\mathrm{dc}}(\mathrm{T})+\sigma_{\mathrm{ac}}(\omega)=\sigma_{\mathrm{dc}}(\mathrm{T})+\mathrm{A}(\mathrm{T}) \omega^{\mathrm{s}}$,

where $\sigma_{\text {total }}$ is the sum of dc and ac conductivity, $\sigma_{\mathrm{dc}}$ is the dc conductivity, $\mathrm{A}(\mathrm{T}) \omega^{\mathrm{s}}$ is the ac conductivity due to the dispersion phenomena occurring in the material, $\mathrm{A}(\mathrm{T})$ is a factor having the unit of conductivity, depends on temperature but not on $\omega$ and $s$ is a power law exponent, which is a temperature and composition dependent quantity and generally varies between 0 and 1 [26]. The exponent $\mathrm{s}$ represents the degree of interaction between mobile ions with the lattice around them and A determines the strength of polarizability [27].

Figure 10 shows Jonscher's plot for all the samples 1 to 5 . 


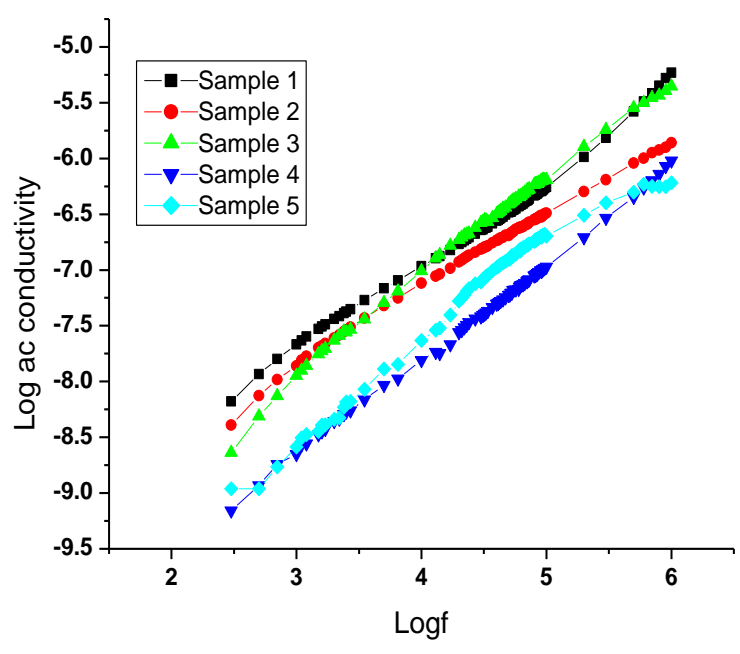

Figure 10. Jonscher's plot

The Jonscher's plot of figure 10 shows highly dispersive behavior for all the samples 1 to 5 due to the existence of ac conductivity. The value of $\mathrm{s}$ and $\mathrm{A}$ can be calculated from the slope and intercept of the plot of figure 10 and listed in table 3 .

Table 3. Jonscher's plot parameters and binding energy for all the samples 1 to 5

\begin{tabular}{|l|l|l|l|l|l|}
\hline $\begin{array}{l}\text { Sample } \\
\text { No }\end{array}$ & $\begin{array}{l}\text { DC } \\
\text { conducti } \\
\text { vity } \\
\left(\sigma_{\mathrm{dc}} \times 10^{-}\right. \\
{ }_{\mathrm{dc}}(\mathrm{S} / \mathrm{m})\end{array}$ & $\begin{array}{l}\mathrm{AC} \\
\text { conductivit } \\
\mathrm{y}\left(\sigma_{\mathrm{ac}} \times 10^{-6}\right) \\
(\mathrm{S} / \mathrm{m})\end{array}$ & $\mathrm{s}$ & $\begin{array}{l}\mathrm{A} \times 10^{-12} \\
\left(\mathrm{~S} \mathrm{~m}^{-1}\right. \\
\left.\mathrm{rad}^{-\mathrm{n}}\right)\end{array}$ & $\begin{array}{l}\text { Binding } \\
\text { energy } \\
\mathrm{W}_{\mathrm{m}} \\
(\mathrm{ev})\end{array}$ \\
\hline 1 & 9.24 & 5.88 & 0.757 & 99.7 & 0.639 \\
\hline 2 & 4.53 & 1.38 & 0.763 & 26.1 & 0.655 \\
\hline 3 & 1.59 & 4.47 & 0.847 & 14.1 & 1.015 \\
\hline 4 & 0.88 & 0.955 & 0.886 & 7.00 & 1.362 \\
\hline 5 & 1.14 & 0.605 & 0.888 & 6.40 & 1.386 \\
\hline
\end{tabular}

From the table 3, one can notice that highest value of strength of polarizability, i.e. the value of $\mathrm{A}$, is obtained for the pure cobalt levo-tartrate crystals of sample 1. As cadmium is added into the pure sample, the value of $\mathrm{A}$ decreases. In moving from sample 2 to 5 , the cadmium content is increased gradually, which results into the gradual decrease in the value of A. Now, dielectric constant is directly related to polarizability of molecules, i.e. greater the polarizability of molecules, higher the dielectric constant of the material. On this basis, the value of strength of polarizability of pure and cadmium added samples are in accordance with the dielectric results.

The value of the exponent $s$ in Jonscher's equation is very useful in the prediction of conduction mechanism in the crystals. The value of exponent $s=0$ indicates frequency independent or dc conduction [26], while $\mathrm{s}=1$ indicates that the interaction between neighboring dipoles is almost negligible [28,29]. The value of $\mathrm{s} \leq 1$ indicates that the hopping motion involved is a translational motion with a sudden hopping [30]. Usually, in case of ionic conductors, the value of exponent can lie between 0.5 and 1 indicating the ideal long range pathways and diffusion limited hopping [31]. In the present case, for all the samples 1 to 5 , it is observed that the value of exponent $\mathrm{s}$ lies between 0.5 and 1 suggesting the above mentioned hopping.

Further, the value of $s$ is found to be low for the pure cobalt levo-tartrate sample 1, which could be attributed to a high rate of successful jumps, results into high dc conductivity [32] as mentioned in table 3. As the cadmium is added into the pure sample 1 and increased in moving from sample 2 to 5 , the value of $\mathrm{s}$ increases respectively. This could be attributed to the decreased rate of successful jumps of ions due to the presence of cadmium at grain position, results into the decreased value of $\mathrm{dc}$ conductivity, respectively. Further, increased interaction between mobile ions with the lattice around them leads to the decrease in the value of exponents [33]. In the present case, the lowest value of exponents for pure cobalt levotartrate sample 1, indicates the highest interaction between the charge carriers and lattice. The gradual increase in the value of exponent $s$ due to the addition of cadmium in the pure sample 1 leads to the decrease in the interaction between the charge carriers and lattice, which may be due to the presence of cadmium at grain position.

In order to get the information about the type of hopping, the composition dependence of exponent $\mathrm{s}$ in hopping over barrier (HOB) model should follow the equation [34] $1-\mathrm{s}$ $=6 \mathrm{k}_{\mathrm{B}} \mathrm{T} / \mathrm{W}_{\mathrm{m}}$, where $\mathrm{k}_{\mathrm{B}}$ is Boltzmann constant, $\mathrm{T}$ is absolute temperature in Kelvin, $\mathrm{s}$ is power law exponent and $\mathrm{W}_{\mathrm{m}}$ is the binding energy, i.e. an energy required to pull out an electron completely from one site to another site. The $\mathrm{W}_{\mathrm{m}}$ values obtained are listed in the table 3 . The results show that the binding energy is minimum for the pure cobalt levo-tartrate sample 1 and then increases as cadmium is added into the pure sample. The increase in binding energy with increase in cadmium content moving from sample 2 to 5 corresponds to the increase in $\mathrm{s}$ parameter. Consequently, the number of free carriers jump over the barrier will be decreased. This result leads to the confirmation of decrease in ac conductivity of cadmium added cobalt levo-tartrate crystals of samples 2 to 5 . This can be verified from the values of ac conductivity at 1 $\mathrm{MHz}$ frequency listed in table 3.

\section{CONCLUSION}

Pure and cadmium mixed cobalt levo-tartrate crystals have been successfully grown by gel method. The impedance measurement shows only one semicircle for pure and cadmium mixed cobalt levo-tartrate crystals suggested the presence of grains and the same was modeled using a $\mathrm{R}$ $\mathrm{CPE}$ parallel circuit. It is also observed that the value of grain resistance increases with the increase in wt\% of cadmium in the pure cobalt levo-tartrate sample. The relaxation time and capacitance have been observed to increase and decrease, respectively with increase in $\mathrm{wt} \%$ of cadmium in the pure cobalt levo-tartrate sample. The Bode plots helped to confirm the presence of the grain effect only. The value of $Z^{\prime}$ increases with the addition of 
cadmium into pure sample of cobalt levo-tartrate indicate decrement of ac conductivity. The Z" relaxation peak is observed for the pure sample of cobalt levo-tartrate and that shifts towards the lower frequency side with increasing height due to an addition of cadmium indicating increment of impedance of cadmium mixed cobalt levotartrate crystals. Though it is beyond the frequency measurement under study, the peaks clearly not appeared. The dielectric properties shows the normal frequency behavior and are explained on the basis of Koops theory and Maxwell-Wagner model. It is observed that the dielectric constant, dielectric loss and ac conductivity decreased with the addition of cadmium. The dielectric constant and dielectric loss decrease with increase in applied frequency due to the inadequacy of electric dipoles to follow the applied field. The prepared samples follow Jonscher's power law. From the values of s obtained from Jonscher's power law suggests ideal long range pathways and diffusion limited hopping. The binding energy increases with increase of cadmium in the pure smple of cobalt levo-tartrate. From the detailed study, it is found that the addition of cadmium and its concentration in cobalt levo-tartrate crystals affects the ac electrical and dielectric properties, which is reflected clearly from the variation of different parameters studied.

\section{ACKNOWLEDGEMENT}

The authors are thankful to UGC for SAP DRS III and DST for FIST and Prof. H. H. Joshi (HOD, Physics) for his keen interest and the author (NHM) is thankful to the authorities of Shree M. M. Science College, Morbi.

\section{REFERENCES}

[1] J. S. Hopwood, A. W. Nicol, "Crystal data for cadmium tartrate pentahydrate”, J. Appl. Cryst.,Vol. 5, pp 437-438, 1972.

[2] I. G. Casella "Electrodeposition of cobalt oxide films from carbonate solutions containing $\mathrm{Co}$ (II) tartrate complexes", $J$. Electroanal. Chem., Vol. 520, Issue.1-2, pp.119-125, 2002.

[3] S. J. Nandre, S. J. Shitole, R. R. Ahire, "FTIR, thermal and optical studies on gel grown cobalt tartrate crystals", J. Nano and Electronic Physics Vol.5 Issue.4, pp.4050(1-5), 2013

[4] V. Mathivanan, M. Haris, T. Prasanyaa, M. Amgalan, "Synthesis and characterization of gel grown cobalt tartrate crystals", Pramana J. Phys., Vol.82, Issue.3, pp. 537-548, 2014.

[5] S. K. Arora, A. Kothari, B. Amin, B. Chudasama, "Synthesis and characterization of cadmium tartrate single crystals", Cryst. Res. Technol., Vol. 42, Issue. 6, pp. 589-594, 2007.

[6] S. K. Arora, A. J. Kothari, R. G. Patel, K. M. Chauhan, B. N. Chudasama, "Optical absorption in gel grown cadmium tartrate single crystals", J. Phys.: Conf. Ser., Vol.28, pp.48-52, 2006.

[7] M. E. Torres, T. Lopez, J. Peraza, J. Stockel, A. C. Yanes, "Structural and dielectric characterization of cadmium tartrate", J. Appl. Phys., Vol.84, Issue.10, pp.5729-5732, 1998.

[8] N. S. Patil, P. A. Chaudhari, D. S. Bhavsar, "Growth of cadmium tartrate crystals by gel technique", Arch. Appl. Sci. Res., Vol.3, Issue.3, pp. 272-278, 2011.

[9] H. K. Henisch, "Crystals growth in gels", Dover Publication, New York., 1993

[10] H. O. Jethva, P. M. Vyas, K. P. Tank, M. J. Joshi, "FTIR and thermal studies of gel grown lead-cadmium mixed levo-tartrate crystals", J. Them. Anal. Calorim., Vol.117, Issue.2, pp. 589$594,2014$.
[11] J. H. Joshi, D. K. Kanchan, M. J. Joshi, H. O. Jethva, K. D. Parikh, "Dielectric relaxation, complex impedance and modulus spectroscopic studies of mix phase rod like cobalt sulfide nanoparticles", Mater. Res. Bull., Vol.93, pp.63-73, 2017.

[12] J. H. Joshi, G. M. Joshi, M. J. Joshi, H. O. Jethva, K. D. Parikh, "Raman, photoluminescence and ac electrical studies of pure and L-serine doped ammonium dihydrogen phosphate single crystals: an understanding of defect chemistry in hydrogen bonding", New J. Chem., Vol.42, pp.17227-17249, 2018.

[13] R. Ranjan, N. Kumar, B. Banarji, R. N. P. Choudhary, "Investigations of impedance and electric modulud properties of $\mathrm{Pb}_{1-\mathrm{x}} \mathrm{Sm}_{\mathrm{x}}\left(\mathrm{Zr}_{0.45} \mathrm{Ti}_{0.55}\right)_{1-\mathrm{x} / 4} \mathrm{O}_{3}$ ceramics", Adv. Mat. Lett., Vol.5, Issue.3, pp.138-142, 2014.

[14] P. R. Das, B. Pati, B. C. Sutar, R. N. P. Choudhury, "Study of structural and electrical properties of a new type of complex tungsten bronze electro ceramics; $\mathrm{Li}_{2} \mathrm{~Pb}_{2} \mathrm{Y}_{2} \mathrm{~W}_{2} \mathrm{Ti}_{4} \mathrm{~V}_{4} \mathrm{O}_{30}$," Journal of Modern Physics, Vol.3, pp.870-880, 2012.

[15] J. C. Anderson, "Dielectrics", Chapman and Hall, London, P. $16,1964$.

[16] R. M. Dabhi, B. Parekh, M. J. Joshi, "Dielectric studies of gel grown zinc tartrate crystals", Indian J. Phys., Vol.79 Issue.5, pp.503-507, 2005.

[17] S. J. Joshi, K. P. Tank, P. M. Vyas, M. J. Joshi, "Structural, FTIR, thermal and dielectric studies of gel grown manganesecopper mixed levo tartrate crystals", J. Cryst. Growth, Vol.401, pp.210-214, 2014.

[18] S. K. Arora, V. Patel, B. Amin, A. Kothari, "Dielectric behavior of strontium tartrate single crystals", Bull. Mater. Sci., Vol.27, Issue.2, pp.141-147, 2004.

[19] C. G. Koops, "On the dispersion of resistivity and dielectric constant of some semiconductors at audio frequencies", Phys. Rev., Vol.83, pp.121-124, 1951.

[20] J. C. Maxwell, "Electricity and Magnetism", Oxford University Press, London 1973.

[21] K. W. Wagner, E. L. Heilman, "The distribution of relaxation times in typical dielectrics", Ann. Phys., Vol.40, pp.818, 1993.

[22] W. K. Lee, B. S. Lim, J. F. Liu, A. S. Nowick, "ac conductivity in ionically conducting crystals and glasses", Solid State Ionics, Vol.831, pp.53-56, 1992.

[23] A. Dutta, C. Bharti, T. P. Sinha, "Dielectric relaxation and ac conductivity study in $\mathrm{SrMg}_{1 / 3} \mathrm{Nb}_{2 / 3} \mathrm{O}_{3}$ ”, Indian J. Eng. Mater. Sci., Vol.15, pp.181-186, 2008.

[24] K. Gohel, D. K. Kanchan, "Ionic conductivity and relaxation studies in PVDF-HFP:PMMA based gel polymer blend epectrolyte with $\mathrm{LiClO}_{4}$ salt", J. Adv. Dielect., Vol.8, pp.185005(1-13), 2018.

[25] A. K. Jonscher, "The universal dielectric response", Nature, Vol.256, pp.673-679, 1977

[26] B. H. Bhat, R. Samad, B. Want, "Dielectric and impedance behavior of neodymium substituted strontium hexaferrite", Appl. Phys. A, Vol.122, pp.810(1-11), 2016.

[27] C. Karthik, K. B. R. Varma, "Dielectric and AC conductivity behavior of $\mathrm{BaBi}_{2} \mathrm{Nb}_{2} \mathrm{O}_{9}$ ceramics", J. Phys. Chem. Solids, Vol.67, pp.2437-2441, 2006.

[28] K. L. Nagi, A. K. Jonscher, C. T. White, "On the origin of the universal dielectric response in condensed matter", Nature, Vol.277, pp.185-189, 1979.

[29] P. Kour, P. Kumar, S. K. Sinha, M. Kar, "Study of dielectric and impedance spectroscopy of La substituted nanocrystalline $\mathrm{Pb}\left(\mathrm{Zr}_{0.52} \mathrm{Ti}_{0.48}\right) \mathrm{O}_{3}$ ceramics", J Mater Sci: Mater Electron, Vol.26, pp.1304-1310, 2015.

[30] K. Funke, "Jump Relaxation in solid electrolytes," Prog. Solid State Chem., Vol.22, pp.111-195, 1993.

[31] K. A. Mauritz, "Dielectric relaxation studies of ion motions in electrolyte-containing perfluorosulfonate ionomers. 4. Long range ion transport”, Macromolecules, Vol.22, pp.4483-4488, 1989.

[32] S. R. Elliott, A. P. Owens, "The diffusion controlled relaxation model for ionic transport in glasses", Philos. Magn., Vol.60, Issue.6, pp.777-792, 1989. 
[33] K. S. Rao, P. M. Krishna, D. M. Prasad, T. S. Latha, C. Satyanarayana, "Low frequency dielectric dispersion studies in ferroelectric ceramics of $\mathrm{Pb}_{0.77} \mathrm{~K}_{0.26} \mathrm{Ti}_{0.25} \mathrm{Nb}_{1.8} \mathrm{O}_{6}$,", Indian $J$. Eng. Mater. Sci., Vol.15, pp.215-223, 2008.

[34] B. K. Chaudhuri, K. Chaudhuri, K. K. Som, "Concentration and frequency dependences of ac conductivity and dielectric constant of Iron-Bismuth oxide glasses", J. Phys. Chem. Solids, Vol.50, Issue.11, pp.1149-1155, 1989.

\section{Author's Profile}

N. H. Manani is a post graduate and M. Phil. from Saurashtra University, Rajkot, Gujarat. He has twenty seven years of experience of teaching Physics to graduate students of various Government colleges. At present, he is working as an Assistant Professor in Physics in M. S. M. Science College, Morbi, Gujarat. He has published more than 25 research papers in National and International Journals of repute, attended and presented papers in more than fifteen national and international conferences and two books on ionosphere.

Dr. H. O. Jethva is a post graduate from Saurashtra University, Rajkot, Gujarat and Doctorate from the same University, has published thirty research papers in International Journals of repute and five books for the undergraduate students of the same university and has experience of teaching physics to undergraduate and post graduate students of twenty four years and at present working as an Associate Professor in the Department of Physics at the same university.

Prof. M. J. Joshi is a post graduate from Saurashtra University, Rajkot, Gujarat and Doctorate from the same University, has published more than one hundred fifty research papers in International Journals of repute and has an experience of teaching physics to post graduate students of thirty one years. He has guided more than fifteen students for Doctorate degree and twenty students for $\mathrm{M}$. Phil. degree. He is active as a subject referee in various journals of International repute. He has refereed more than sixty five Ph. D. Thesis. At present he is working as Head and Professor of Physics in the same university. 